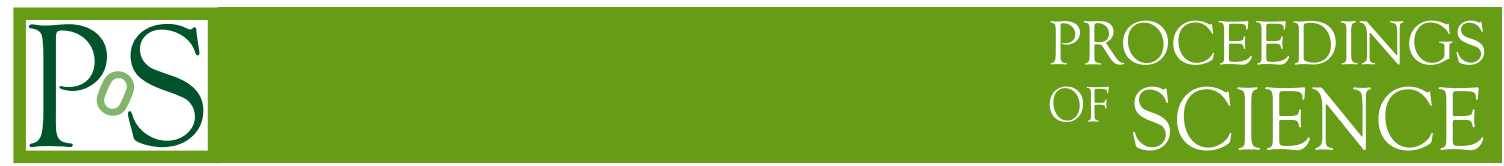

\title{
Probing New Physics with heavy hadron decays
}

\author{
Pietro Colangelo, ${ }^{a}$ Fulvia De Fazio ${ }^{a, *}$ and Francesco Loparco ${ }^{a, b}$ \\ ${ }^{a}$ Istituto Nazionale di Fisica Nucleare, Sezione di Bari \\ Via Orabona 4, I-70126 Bari, Italy \\ ${ }^{b}$ Universitá degli Studi di Bari \\ Via Orabona 4, I-70126 Bari, Italy \\ E-mail: pietro.colangelo@ba.infn.it, fulvia.defazio@ba.infn.it, \\ francesco. loparco1@ba.infn. it
}

The increasing number of flavour anomalies calls for the investigation of new processes where tensions similar to the observed ones could emerge. Observables sensitive to physics beyond the Standard Model need to be identified. We discuss the inclusive semileptonic decays of polarized beauty baryons, computed through the heavy quark expansion at $O\left(1 / m_{b}^{3}\right)$ and at the leading order in $\alpha_{s}$. We account for New Physics interactions in a model-independent way, extending the Standard Model $b \rightarrow U \ell \bar{v}_{\ell}$ low energy Hamiltonian (with $U=u, c$ and $\ell=e, \mu, \tau$ ) with the inclusion of the full set of $\mathrm{D}=6$ semileptonic operators with left-handed neutrinos. We identify a set of promising observables, the study of which can be included in the physics programmes of future facilities, such as FCC-ee.

\footnotetext{
*** The European Physical Society Conference on High Energy Physics (EPS-HEP2021), *** *** 26-30 July $2021 * * *$

*** Online conference, jointly organized by Universität Hamburg and the research center DESY ***
}

\footnotetext{
${ }^{*}$ Speaker
} 


\section{Introduction}

In the searches for signals of New Physics (NP), deviations in a number of observables with respect to the Standard Model (SM) predictions have recently been detected. They constitute the so-called flavour anomalies, and show up in selected tree-level and loop-induced decays of $B, B_{s}$ and $B_{c}$ mesons [1]. In particular, hints of lepton flavour universality (LFU) violations have been collected. The observed deviations call for new investigations, in particular focused on other heavy hadron decay processes. Here, we analyze the inclusive semileptonic beauty baryon decays [2], for which the nonperturbative effects of strong interactions can be systematically treated exploiting an expansion in the inverse heavy quark mass [3, 4].

Even though the formalism holds for a generic baryon comprising a heavy quark, we focus on inclusive $\Lambda_{b} \rightarrow X_{c, u} \ell^{-} \bar{v}_{\ell}$ decays, performing the heavy quark mass expansion (HQE) at $O\left(1 / m_{Q}^{3}\right)$. At each order in the expansion, a number of hadronic matrix elements is required; they are parametrized in terms of basic non perturbative quantities. Our main result is the derivation of the baryon matrix elements at $O\left(1 / m_{Q}^{3}\right)$ in the case of a polarized baryon. The result is applied to derive the fully differential decay rate for $\Lambda_{b} \rightarrow X_{c, u} \ell^{-} \bar{v}_{\ell}$ both in the SM and in an extension of the SM effective weak Hamiltonian comprising vector, scalar, pseudoscalar, tensor and axial operators. Other studies extending the effective Hamiltonian in a similar way are in [5-11].

At LHC the $\Lambda_{b}$ is produced unpolarized [12-15] since the $b$ quark mainly comes from QCD processes. A sizable longitudinal $\Lambda_{b}$ polarization is expected for $b$ quarks produced in $Z$ and top quark decays [16-18]. The investigations of effects beyond the Standard Model (BSM) in the polarized case must be optimized in such an experimental environment. Our analysis and results are presented below.

\section{Generalized effective weak Hamiltonian}

We consider a beauty baryon $H_{b}$ with spin $s$. The inclusive semileptonic $H_{b}(p, s) \rightarrow$ $X_{U}\left(p_{X}\right) \ell^{-}\left(p_{\ell}\right) \bar{v}_{\ell}\left(p_{v}\right)$ decays $(U=u, c)$, induced by the $b \rightarrow u, c$ transitions, are described by the general low-energy Hamiltonian, which extends the SM one:

$$
\begin{aligned}
& H_{\mathrm{eff}}^{b \rightarrow U \ell v}=\frac{G_{F}}{\sqrt{2}} V_{U b} \\
& {\left[\left(1+\epsilon_{V}^{\ell}\right)\left(\bar{U} \gamma_{\mu}\left(1-\gamma_{5}\right) b\right)\left(\bar{\ell} \gamma^{\mu}\left(1-\gamma_{5}\right) v_{\ell}\right)+\epsilon_{S}^{\ell}(\bar{U} b)\left(\bar{\ell}\left(1-\gamma_{5}\right) v_{\ell}\right)+\epsilon_{P}^{\ell}\left(\bar{U} \gamma_{5} b\right)\left(\bar{\ell}\left(1-\gamma_{5}\right) v_{\ell}\right)\right.} \\
& \left.+\epsilon_{T}^{\ell}\left(\bar{U} \sigma_{\mu \nu}\left(1-\gamma_{5}\right) b\right)\left(\bar{\ell} \sigma^{\mu \nu}\left(1-\gamma_{5}\right) v_{\ell}\right)+\epsilon_{R}^{\ell}\left(\bar{U} \gamma_{\mu}\left(1+\gamma_{5}\right) b\right)\left(\bar{\ell} \gamma^{\mu}\left(1-\gamma_{5}\right) v_{\ell}\right)\right]+ \text { h.c. . }
\end{aligned}
$$

$\epsilon_{V, S, P, T, R}^{\ell}$ are complex and lepton-flavour dependent coefficients. Only left-handed neutrinos are considered, and $V_{U b}$ is the relevant Cabibbo-Kobayashi-Maskawa (CKM) matrix element. The Hamiltonian can be written as

$$
H_{\mathrm{eff}}^{b \rightarrow U \ell v}=\frac{G_{F}}{\sqrt{2}} V_{U b} \sum_{i=1}^{5} C_{i}^{\ell} J_{M}^{(i)} L^{(i) M}+\text { h.c. },
$$

with $C_{1}^{\ell}=\left(1+\epsilon_{V}^{\ell}\right)$ and $C_{2,3,4,5}^{\ell}=\epsilon_{S, P, T, R}^{\ell} . J_{M}^{(i)}\left(L^{(i) M}\right)$ is the hadronic (leptonic) current in each operator, $M$ are set of Lorentz indices contracted between $J$ and $L$. The SM expression is recovered for $i=1$ and $\epsilon_{V, S, P, T, R}^{\ell}=0$. We keep $m_{\ell} \neq 0$ for all leptons $\ell=e, \mu, \tau$. 


\section{Inclusive decay width}

The $H_{b}$ inclusive semileptonic differential decay width can be written as

$$
d \Gamma=d \Sigma \frac{G_{F}^{2}\left|V_{U b}\right|^{2}}{4 m_{H}} \sum_{i, j} C_{i}^{*} C_{j}\left(W^{i j}\right)_{M N}\left(L^{i j}\right)^{M N} .
$$

$G_{F}$ is the Fermi constant, $q=p_{\ell}+p_{v}$ is the lepton pair momentum, and $d \Sigma$ is the phasespace element $d \Sigma=(2 \pi) d^{4} q \delta^{4}\left(q-p_{\ell}-p_{\nu}\right)\left[d p_{\ell}\right]\left[d p_{\nu}\right]$, with $[d p]=\frac{d^{3} p}{(2 \pi)^{3} 2 p^{0}}$. The leptonic tensor is $\left(L^{i j}\right)^{M N}=L^{(i) \dagger M} L^{(j) N}$. On the basis of the optical theorem, the hadronic tensor $\left(W^{i j}\right)_{M N}=\frac{1}{\pi} \operatorname{Im}\left(T^{i j}\right)_{M N}$ is given in terms of the forward amplitude

$$
\left(T^{i j}\right)_{M N}=i \int d^{4} x e^{-i q \cdot x}\left\langle H_{b}(p, s)\left|T\left[J_{M}^{(i) \dagger}(x) J_{N}^{(j)}(0)\right]\right| H_{b}(p, s)\right\rangle .
$$

The hadron momentum $p=m_{b} v+k$ is written in terms of the four-velocity $v$, the heavy quark mass $m_{b}$ and a residual momentum $k$ of order $\Lambda_{Q C D}$. The QCD $b$ quark field $b(x)$ is redefined as $b(x)=e^{-i m_{b} v \cdot x} b_{v}(x)$, with $b_{v}(x)$ satisfying the equation $b_{v}(x)=\left(P_{+}+\frac{i D}{2 m_{b}}\right) b_{v}(x)$, with $P_{+}=\frac{1+\not}{2}$ the velocity projector. Introducing $p_{X}=m_{b} v+k-q$ we have:

$$
\left(T^{i j}\right)_{M N}=\left\langle H_{b}(v, s)\left|\bar{b}_{v}(0) \Gamma_{M}^{(i) \dagger} S_{U}\left(p_{X}\right) \Gamma_{N}^{(j)} b_{v}(0)\right| H_{b}(v, s)\right\rangle,
$$

with $S_{U}\left(p_{X}\right)$ the $U$ quark propagator. The heavy quark expansion in powers of $m_{b}^{-1}$ is carried out $[3,4]$. It is obtained replacing $k \rightarrow i D$ ( $D$ is the QCD covariant derivative) and expanding $S_{U}\left(p_{X}\right)=S_{U}^{(0)}-S_{U}^{(0)}(i D) S_{U}^{(0)}+S_{U}^{(0)}(i D) S_{U}^{(0)}(i D) S_{U}^{(0)}+\ldots$ where $S_{U}^{(0)}=\frac{1}{m_{b} \not b-\not q-m_{U}}$. Writing $p_{U}=m_{b} v-q, \mathcal{P}=\left(p_{U}+m_{U}\right)$ and $\Delta_{0}=p_{U}^{2}-m_{U}^{2}$, the expansion at order $1 / m_{b}^{3}$ reads:

$$
\begin{aligned}
\frac{1}{\pi} \operatorname{Im}\left(T^{i j}\right)_{M N}= & \frac{1}{\pi} \operatorname{Im} \frac{1}{\Delta_{0}}\left\langle H_{b}(v, s)\left|\bar{b}_{v}\left[\Gamma_{M}^{(i) \dagger} \mathcal{P} \Gamma_{N}^{(j)}\right] b_{v}\right| H_{b}(v, s)\right\rangle+ \\
& -\frac{1}{\pi} \operatorname{Im} \frac{1}{\Delta_{0}^{2}}\left\langle H_{b}(v, s)\left|\bar{b}_{v}\left[\Gamma_{M}^{(i) \dagger} \mathcal{P} \gamma^{\mu_{1}} \mathcal{P} \Gamma_{N}^{(j)}\right]\left(i D_{\mu_{1}}\right) b_{v}\right| H_{b}(v, s)\right\rangle+ \\
& +\frac{1}{\pi} \operatorname{Im} \frac{1}{\Delta_{0}^{3}}\left\langle H_{b}(v, s)\left|\bar{b}_{v}\left[\Gamma_{M}^{(i) \dagger} \mathcal{P} \gamma^{\mu_{1}} \mathcal{P} \gamma^{\mu_{2}} \mathcal{P} \Gamma_{N}^{(j)}\right]\left(i D_{\mu_{1}}\right)\left(i D_{\mu_{2}}\right) b_{v}\right| H_{b}(v, s)\right\rangle+ \\
& -\frac{1}{\pi} \operatorname{Im} \frac{1}{\Delta_{0}^{4}}\left\langle H_{b}(v, s)\left|\bar{b}_{v}\left[\Gamma_{M}^{(i) \dagger} \mathcal{P} \gamma^{\mu_{1}} \mathcal{P} \gamma^{\mu_{2}} \mathcal{P} \gamma^{\mu_{3}} \mathcal{P} \Gamma_{N}^{(j)}\right]\left(i D_{\mu_{1}}\right)\left(i D_{\mu_{2}}\right)\left(i D_{\mu_{3}}\right) b_{v}\right| H_{b}(v, s)\right\rangle .
\end{aligned}
$$

The expression (6) involves the hadronic matrix elements

$$
\mathcal{M}_{\mu_{1} \ldots \mu_{n}}=\left\langle H_{b}(v, s)\left|\left(\bar{b}_{v}\right)_{a}\left(i D_{\mu_{1}}\right) \ldots\left(i D_{\mu_{n}}\right)\left(b_{v}\right)_{b}\right| H_{b}(v, s)\right\rangle,
$$

with $a, b$ Dirac indices. The matrix elements can be written in terms of nonperturbative parameters, the number of which increases with the order of the expansion. At $O\left(1 / m_{b}^{3}\right)$ the required ones are:

$$
\begin{aligned}
\left\langle H_{b}(v, s)\left|\bar{b}_{v}(i D)^{2} b_{v}\right| H_{b}(v, s)\right\rangle & =-2 m_{H} \hat{\mu}_{\pi}^{2} \\
\left\langle H_{b}(v, s)\left|\bar{b}_{v}\left(i D_{\mu}\right)\left(i D_{v}\right)\left(-i \sigma^{\mu v}\right) b_{v}\right| H_{b}(v, s)\right\rangle & =2 m_{H} \hat{\mu}_{G}^{2} \\
\left\langle H_{b}(v, s)\left|\bar{b}_{v}\left(i D_{\mu}\right)(i v \cdot D)\left(i D^{\mu}\right) b_{v}\right| H_{b}(v, s)\right\rangle & =2 m_{H} \hat{\rho}_{D}^{3} \\
\left\langle H_{b}(v, s)\left|\bar{b}_{v}\left(i D_{\mu}\right)(i v \cdot D)\left(i D_{v}\right)\left(-i \sigma^{\mu v}\right) b_{v}\right| H_{b}(v, s)\right\rangle & =2 m_{H} \hat{\rho}_{L S}^{3} .
\end{aligned}
$$


A method to compute $\mathcal{M}_{\mu_{1} \ldots \mu_{n}}$ is proposed in [19,20]. In the case of baryons the dependence on the spin $s_{\mu}$ in (7) must be kept. In [2] $\mathcal{M}_{\mu_{1} \ldots \mu_{n}}$ have been derived at order $1 / m_{b}^{3}$ for a polarized baryon, considering all operators in (1). This extends previous results in $[9,11,21-23]$.

From the expressions of the matrix elements $\mathcal{M}_{\mu_{1} \ldots \mu_{n}}$ the hadronic tensor can be computed and expanded in Lorentz structures which depend on $v, q$ and $s$. The results for the SM and for the effective Hamiltonian Eq. (1) are collected in [2]. The four-fold differential decay rate for the $H_{b}(v, s) \rightarrow X\left(p_{X}\right) \ell^{-}\left(p_{\ell}\right) \bar{v}_{\ell}\left(p_{v}\right)$ transition reads

$$
\frac{d^{4} \Gamma}{d q^{2} d(v \cdot q) d E_{\ell} d \cos \theta_{P}}=\frac{G_{F}^{2}\left|V_{U b}\right|^{2}}{32(2 \pi)^{3} m_{H}} \sum_{i, j} C_{i}^{*} C_{j} \frac{1}{\pi} \operatorname{Im}\left(T^{i j}\right)_{M N}\left(L^{i j}\right)^{M N}
$$

with $p_{\ell}=\left(E_{\ell}, \vec{p}_{\ell}\right) . \theta_{P}$ the angle between $\vec{p}_{\ell}$ and $\vec{s}$ in the $H_{b}$ rest frame. Double and single decay distributions are obtained integrating (12) over the phase-space [24]. Performing all integrations, the full decay width can be written as:

$$
\Gamma\left(H_{b} \rightarrow X \ell^{-} \bar{v}_{\ell}\right)=\Gamma_{b} \sum_{i}\left\{C_{0}^{(i)}+\frac{\mu_{\pi}^{2}}{m_{b}^{2}} C_{\mu_{\pi}^{2}}^{(i)}+\frac{\mu_{\pi}^{2}}{m_{b}^{2}} C_{\mu_{G}^{2}}^{(i)}+\frac{\rho_{D}^{3}}{m_{b}^{3}} C_{\rho_{D}^{3}}^{(i)}+\frac{\rho_{L S}^{3}}{m_{b}^{3}} C_{\rho_{L S}^{3}}^{(i)}\right\},
$$

with $\Gamma_{b}=\frac{G_{F}^{2} m_{b}^{5} V_{U b}^{2}}{192 \pi^{3}}$. The index $i$ runs over the contribution of the various operators and of their interferences. The coefficients $C^{(i)}$ depend on the NP couplings in (1) and can be found in [2].

The OPE breaks down in the endpoint region of the spectra, where singularities appear. They require to be resummed in a $H_{b}$ shape function, and the convolution with such a function smears the spectra at the endpoint. We have not considered the effects of the baryon shape function, which is not known at present. Perturbative QCD corrections are also not included: in the SM various corrections have been computed [25-30].

\section{Results for $\Lambda_{b} \rightarrow X_{c, u} \ell \bar{v}_{\ell}$}

We collect in this Section some results obtained for observables in $\Lambda_{b} \rightarrow X_{u, c} \ell^{-} \bar{v}_{\ell}$. We refer to [2] for the input parameters. For the couplings $\epsilon_{V, S, P, T, R}^{\ell}$ in (1), for $U=u$ we use the ranges fixed in [31]. For $U=c$ we fix three NP benchmark points, set in [6] and [32].

In the SM, using $\left|V_{c b}\right|=0.042,\left|V_{u b}\right|=0.0037$ and $\tau_{\Lambda_{b}}=(1.471 \pm 0.009) \times 10^{-12} \mathrm{~s}$ [33], we obtain: $\mathcal{B}\left(\Lambda_{b} \rightarrow X_{c} \mu \bar{v}_{\mu}\right)=11.0 \times 10^{-2}, \mathcal{B}\left(\Lambda_{b} \rightarrow X_{c} \tau \bar{v}_{\tau}\right)=2.4 \times 10^{-2}, \mathcal{B}\left(\Lambda_{b} \rightarrow X_{u} \mu \bar{v}_{\mu}\right)=$ $11.65 \times 10^{-4}$ and $\mathcal{B}\left(\Lambda_{b} \rightarrow X_{u} \tau \bar{v}_{\tau}\right)=2.75 \times 10^{-4}$. For comparison, the available measurements are $\mathcal{B}\left(\Lambda_{b} \rightarrow \Lambda_{c} \ell^{-} \bar{v}_{\ell}+\right.$ anything $)=(10.9 \pm 2.2) \times 10^{-2} \ell=e, \mu$, and $\mathcal{B}\left(\Lambda_{b} \rightarrow p \mu^{-} \bar{v}\right)=$ $(4.1 \pm 1.0) \times 10^{-4}[33]$.

Among the various interesting observables, for polarized $\Lambda_{b}$ we mention here the distribution $\frac{d \Gamma\left(\Lambda_{b} \rightarrow X_{U} \ell \bar{v}_{\ell}\right)}{d \cos \theta_{P}}=A_{\ell}^{U}+B_{\ell}^{U} \cos \theta_{P}$. In the ratio $R_{\Lambda_{b}}\left(X_{U}\right)=\frac{\Gamma\left(\Lambda_{b} \rightarrow X_{U} \tau \bar{v}_{\tau}\right)}{\Gamma\left(\Lambda_{b} \rightarrow X_{U} \mu \bar{v}_{\mu}\right)}=\frac{A_{\tau}^{U}}{A_{\mu}^{U}}$ several theoretical uncertainties cancel out. The predictions in SM and in NP at the chosen benchmark points are: $R_{\Lambda_{b}}\left(X_{u}\right)^{S M}=0.234, R_{\Lambda_{b}}\left(X_{u}\right)^{N P}=0.238, R_{\Lambda_{b}}\left(X_{c}\right)^{S M}=0.214, R_{\Lambda_{b}}\left(X_{c}\right)^{N P}=0.240$, at the leading order in $\alpha_{s}$. Also the ratio $R_{S}^{U}=B_{\tau}^{U} / B_{\mu}^{U}$ is sensitive to NP. In SM we find: $R_{S}^{c}=0.1$ and $R_{S}^{u}=0.08$. To study the correlation between $R_{\Lambda_{b}}\left(X_{U}\right)$ and $R_{S}^{U}$ we consider, as an example, 


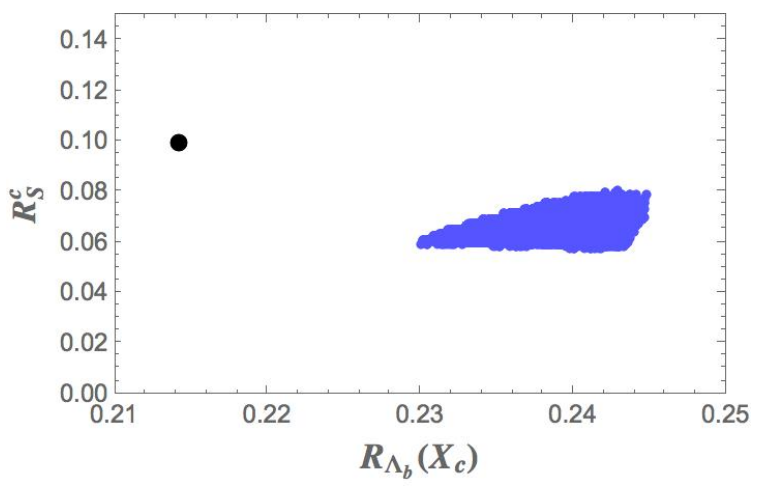

Figure 1: correlation between $R_{\Lambda_{b}}\left(X_{c}\right)$ and $R_{S}^{c}$. The dot corresponds to SM, the broad region to NP.

the generalized effective Hamiltonian extended only with the tensor operator. The correlation plot in Fig. 1 shows that, although experimentally challenging, the measurement of the two ratios discriminates SM from NP.

\section{Conclusions}

We have described the calculation of the inclusive semileptonic decay width of a polarized heavy hadron at order $O\left(1 / \mathrm{m}_{b}^{3}\right)$ in the HQE, at leading order in $\alpha_{s}$ and for non vanishing charged lepton mass. NP is considered extending the SM effective Hamiltonian including the full set of $D=6$ semileptonic operators. Among the various result, the correlation in Fig. 1 shows the discriminating power between SM and NP which can be obtained by the analysis of inclusive polarized $\Lambda_{b}$ semileptonic modes.

\section{Acknowledgments}

This study has been carried out within the INFN project (Iniziativa Specifica) QFT-HEP.

\section{References}

[1] M. Algueró et al., $\boldsymbol{b} \rightarrow \boldsymbol{s} \boldsymbol{\ell \ell}$ global fits after Moriond 2021 results, in 55th Rencontres de Moriond on QCD and High Energy Interactions, 4, 2021. arXiv: 2104.08921.

[2] P. Colangelo, F. De Fazio, and F. Loparco, Inclusive semileptonic $\Lambda_{b}$ decays in the Standard Model and beyond, JHEP 11 (2020) 032, [arXiv: 2006. 13759].

[3] J. Chay, H. Georgi, and B. Grinstein, Lepton energy distributions in heavy meson decays from QCD, Phys. Lett. B 247 (1990) 399-405.

[4] I. I. Bigi et al., QCD predictions for lepton spectra in inclusive heavy flavor decays, Phys. Rev. Lett. 71 (1993) 496-499, [hep-ph/9304225]. 
[5] P. Biancofiore, P. Colangelo, and F. De Fazio, On the anomalous enhancement observed in $B \rightarrow D^{(*)} \tau \bar{v}_{\tau}$ decays, Phys. Rev. $\mathbf{D 8 7}$ (2013) 074010, [arXiv: 1302. 1042].

[6] P. Colangelo and F. De Fazio, Scrutinizing $\bar{B} \rightarrow D^{*}(D \pi) \ell^{-} \bar{v}_{\ell}$ and $\bar{B} \rightarrow D^{*}(D \gamma) \ell^{-} \bar{v}_{\ell}$ in search of new physics footprints, JHEP 06 (2018) 082, [arXiv: 1801 . 10468].

[7] S. Bhattacharya et al., $b \rightarrow c \tau v_{\tau}$ Decays: a catalogue to compare, constrain, and correlate new physics effects, Eur. Phys. J. C 79 (2019) 268, [arXiv: 1805 . 08222].

[8] P. Colangelo and F. De Fazio, Tension in the inclusive versus exclusive determinations of $\left|V_{c b}\right|$ : a possible role of new physics, Phys. Rev. D95 (2017) 011701, [arXiv: 1611.07387].

[9] T. Mannel, A. V. Rusov, and F. Shahriaran, Inclusive semitauonic B decays to order $O\left(\Lambda_{Q C D}^{3} / m_{b}^{3}\right)$, Nucl. Phys. B 921 (2017) 211-224, [arXiv: 1702.01089].

[10] S. Kamali, A. Rashed, and A. Datta, New physics in inclusive $B \rightarrow X_{c} \ell \bar{v}$ decay in light of $R\left(D^{(*)}\right)$ measurements, Phys. Rev. $D 97$ (2018) 095034, [arXiv: 1801.08259 ].

[11] S. Kamali, New physics in inclusive semileptonic B decays including nonperturbative corrections, Int. J. Mod. Phys. A34 (2019) 1950036, [arXiv: 1811.07393].

[12] LHCb Collaboration, R. Aaij et al., Measurements of the $\Lambda_{b}^{0} \rightarrow J / \psi \Lambda$ decay amplitudes and the $\Lambda_{b}^{0}$ polarisation in pp collisions at $\sqrt{s}=7$ TeV, Phys. Lett. B 724 (2013) 27-35, [arXiv: 1302.5578].

[13] ATLAS Collaboration, G. Aad et al., Measurement of the parity-violating asymmetry parameter $\alpha_{b}$ and the helicity amplitudes for the decay $\Lambda_{b}^{0} \rightarrow J / \psi+\Lambda^{0}$ with the ATLAS detector, Phys. Rev. D 89 (2014) 092009, [arXiv: 1404 . 1071].

[14] CMS Collaboration, A. M. Sirunyan et al., Measurement of the $\Lambda_{b}$ polarization and angular parameters in $\Lambda_{b} \rightarrow J / \psi \Lambda$ decays from pp collisions at $\sqrt{s}=7$ and 8 TeV, Phys. Rev. D 97 (2018) 072010, [arXiv: 1802.04867$]$.

[15] LHCb Collaboration, R. Aaij et al., Measurement of the $\Lambda_{b}^{0} \rightarrow J / \psi \Lambda$ angular distribution and the $\Lambda_{b}^{0}$ polarisation in pp collisions, arXiv: 2004.10563.

[16] ALEPH Collaboration, D. Buskulic et al., Measurement of the $\Lambda_{b}$ polarization in $Z$ decays, Phys. Lett. B 365 (1996) 437 - 447.

[17] OPAL Collaboration, G. Abbiendi et al., Measurement of the average polarization of $b$ baryons in hadronic $Z^{0}$ decays, Phys. Lett. B 444 (1998) 539-554, [hep-ex/9808006].

[18] DELPHI Collaboration, P. Abreu et al., $\Lambda_{b}$ polarization in $Z^{0}$ decays at LEP, Phys. Lett. $B$ 474 (2000) $205-222$.

[19] B. M. Dassinger, T. Mannel, and S. Turczyk, Inclusive semi-leptonic B decays to order $1 /$ $m(b) * * 4$, JHEP 03 (2007) 087, [hep-ph/0611168]. 
[20] T. Mannel, S. Turczyk, and N. Uraltsev, Higher Order Power Corrections in Inclusive B Decays, JHEP 11 (2010) 109, [arXiv: 1009.4622].

[21] Y. Grossman and Z. Ligeti, The Inclusive $\bar{B} \rightarrow \tau \bar{v} X$ decay in two Higgs doublet models, Phys. Lett. B 332 (1994) 373-380, [hep-ph/9403376].

[22] A. V. Manohar and M. B. Wise, Inclusive semileptonic B and polarized Lambda(b) decays from QCD, Phys. Rev. D49 (1994) 1310-1329, [hep-ph/9308246].

[23] S. Balk, J. Korner, and D. Pirjol, Inclusive semileptonic decays of polarized $\Lambda_{b}$ baryons into polarized $\tau$ leptons, Eur. Phys. J. C 1 (1998) 221-233, [hep-ph/9703344].

[24] M. Jezabek and L. Motyka, Perturbative QCD corrections to inclusive lepton distributions from semileptonic $b \rightarrow c \tau \bar{v}_{\tau}$ decays, Acta Phys. Polon. B 27 (1996) 3603-3613, [hep-ph/9609352].

[25] A. Czarnecki, M. Jezabek, and J. H. Kuhn, Radiative corrections to $b \rightarrow c \tau \bar{v}_{\tau}$, Phys. Lett. B 346 (1995) 335-341, [hep-ph/9411282].

[26] M. Jezabek and L. Motyka, Tau lepton distributions in semileptonic B decays, Nucl. Phys. B 501 (1997) 207-223, [hep-ph/9701358].

[27] F. De Fazio and M. Neubert, $B \rightarrow X_{u} \ell \bar{v}_{\ell}$ decay distributions to order $\alpha_{s}$, JHEP 06 (1999) 017, [hep-ph/9905351].

[28] M. Trott, Improving extractions of $|V(c b)|$ and $m(b)$ from the hadronic invariant mass moments of semileptonic inclusive B decay, Phys. Rev. D 70 (2004) 073003, [hep-ph/0402120].

[29] V. Aquila, P. Gambino, G. Ridolfi, and N. Uraltsev, Perturbative corrections to semileptonic b decay distributions, Nucl. Phys. B 719 (2005) 77-102, [hep-ph/0503083].

[30] A. Alberti et al., Precision Determination of the Cabibbo-Kobayashi-Maskawa Element $V_{c b}$, Phys. Rev. Lett. 114 (2015) 061802, [arXiv: 1411.6560].

[31] P. Colangelo, F. De Fazio, and F. Loparco, Probing New Physics with $\bar{B} \rightarrow \rho(770) \ell^{-} \bar{v}_{\ell}$ and $\bar{B} \rightarrow a_{1}(1260) \ell^{-} \bar{v}_{\ell}$, Phys. Rev. D 100 (2019) 075037, [arXiv: 1906.07068].

[32] R.-X. Shi et al., Revisiting the new-physics interpretation of the $b \rightarrow c \tau v$ data, JHEP 12 (2019) 065, [arXiv: 1905.08498].

[33] Particle Data Group Collaboration, P. A. Zyla et al., Review of Particle Physics, Prog. Theor. Exp. Phys. 2020 (2020) 083501. 